\title{
CORRELATION-DRIVEN METAL-INSULATOR TRANSITIONS
}

\author{
J.M. HONIG \\ Department of Chemistry, Purdue University, West Lafayette, 47907-1393, USA
}

The effect of correlation-driven electronic transitions are described for the $\mathrm{V}_{2} \mathrm{O}_{3}, \mathrm{NiS}_{2-x} \mathrm{Se}_{x}$, and $\mathrm{Fe}_{3} \mathrm{O}_{4}$ systems. The various tranformations can all be rationalized in terms of elementary concepts pertaining to the MottHubbard intraatomic electronic interactions or in terms of an order-disorder formalism involving Coulomb interactions among electrons on adjacent sites. Attention is directed to some outstanding issues that require further resolution.

PACS numbers: $71.30 .+\mathrm{h}, 71.45 . \mathrm{Gm}$

\section{Introduction}

In this paper we briefly review the properties of $\mathrm{V}_{2} \mathrm{O}_{3}, \mathrm{NiS}_{2}-\mathrm{NiSe}_{2}$, and $\mathrm{Fe}_{3} \mathrm{O}_{4}$ that represent a class of materials undergoing metal-insulator transitions as a result of electron correlation effects. For more complete accounts, the reader is referred to several review articles $[1,2]$.

\section{The $\mathrm{V}_{2} \mathrm{O}_{3}$ system}

$\mathrm{V}_{2} \mathrm{O}_{3}$ is a particularly interesting example that illustrates how doping with aliovalent cations, application of hydrostatic pressure, and introducing cation vacancies via oxygen excess, can drastically alter the electrical characteristics and related physical properties of the parent $\mathrm{V}_{2} \mathrm{O}_{3}$ compound.

In Fig. 1 we show the effects of $\mathrm{Cr}$ doping [3]. In a limited range of compositions $x$ in $\left(\mathrm{V}_{1-x} \mathrm{Cr}_{x}\right)_{2} \mathrm{O}_{3}$ the alloys show three back-to-back metal-insulator transitions (MIT) that separate a low temperature antiferromagnetic insulating (AFI) phase from a paramagnetic metallic $\left(\mathrm{PM}^{\prime}\right)$ regime closer to room temperature, that in turn switches discontinuously to a paramagnetic insulating (PI) phase, and then changes gradually into a second paramagnetic metallic $\left(\mathrm{PM}^{\prime}\right)$ phase at highest temperatures. The PM-AFI transformation is driven in part by magnetic ordering effects; additionally, the lattice distorts from the corundum (PM) to the monoclinic (AFI) configuration. The reentrant metallic feature, i.e., the sequence of transitions $\mathrm{PM}-\mathrm{PI}-\mathrm{PM}^{\prime}$ with rising temperature $T$ requires explanation. For 


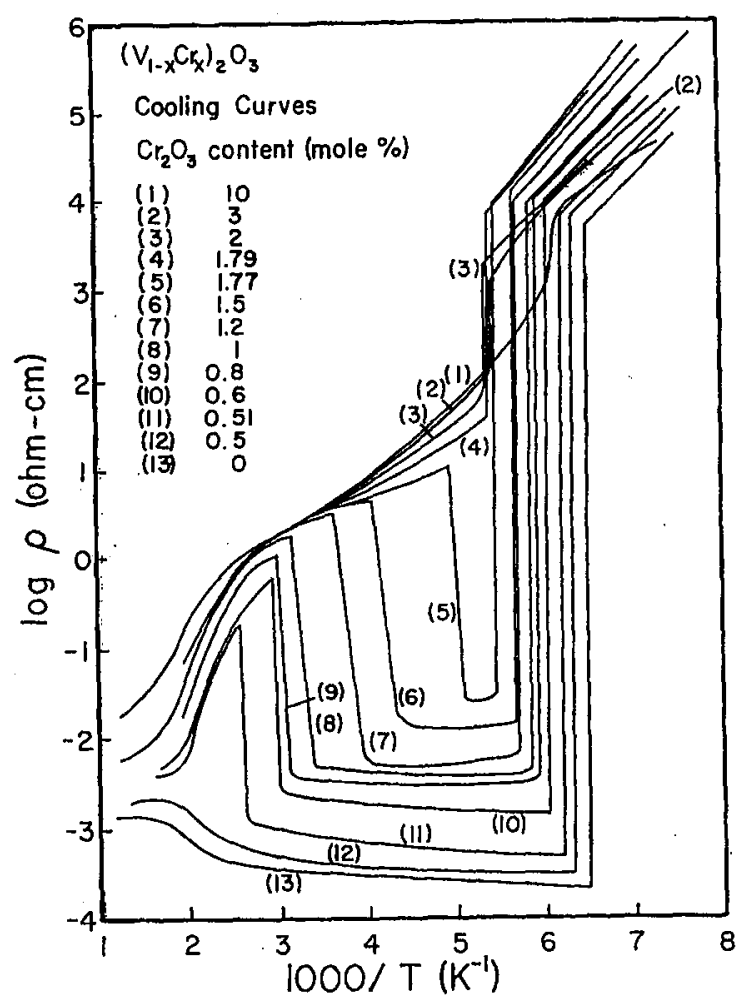

Fig. 1. Resisitivity of $\left(\mathrm{V}_{1-x} \mathrm{Cr}_{x}\right)_{2} \mathrm{O}_{3}$ single crystals for indicated $x$ values as a function of temperature.

completeness, note that incorporation of $\mathrm{Ti}$, or excess $\mathrm{O}$ [4], or application of pressure [5] ultimately suppresses the insulating phase altogether; the corresponding alloys are poor metals down to the cryogenic temperature range where the PM phase changes to an antiferromagnetic metal (AFM) [6].

The experimental information immediately raises two interrelated points: one must show how these materials can undergo drastic electronic rearrangements on the scale of 1-2 eV in response to thermal inputs $k_{\mathrm{B}} T$ of the order of $10 \mathrm{meV}$; also, all of the different phase transitions (as well as their absence) must be accounted for.

As a step in that direction, we offer a very simplistic explanation that must be supplemented by a far more sophisticated rationalization offered in the literature [7]. We first establish that electron correlation effects do govern the electronic properties of the $\mathrm{V}_{2} \mathrm{O}_{3}$ system. Three experimental manifestations of electron correlations are generally invoked: The first is the temperature dependence of resistivity $\rho$ in the cryogenic temperature $(T)$ range: $\rho=\rho_{0}+A T^{2}$. This manifests the so-called Baber-Pomeranchuk scattering effects, due to electron interactions. It has been verified [5] that metallic $\mathrm{V}_{2} \mathrm{O}_{3}$ under hydrostatic pressures does follow this law. Second, the electronic contribution to the heat capacity follows the linear- 
ity expression $C / N=\gamma T=\gamma_{0} /\left(1-I^{2}\right) T$, where $\gamma_{0}$ is the Sommerfeld constant for free electrons, $I \equiv U / U_{\mathrm{c}}$, and $N$ is the number of lattice sites; $U$ is the Coulomb repulsion potential between two electrons residing on the same lattice site, and $U_{c}$ is the critical value beyond which electron localization takes place. Clearly, as $U \rightarrow U_{\mathrm{c}}$ the value of $\gamma$ can become very large. This has been shown to be the case for $\mathrm{V}_{2} \mathrm{O}_{3}$ rendered metallic by doping or under pressure; the experimental $\gamma$ values are at least one order of magnitude larger than those found in conventional metals. Third, at low $T$ the Pauli paramagnetic susceptibility for a correlated electron gas is given by $\chi=\chi_{0}\left(1-B T^{2}\right) /\left(1-I_{2}\right) S$, where $\chi_{0}$ is the susceptibility for the free electron gas and $S=1-f U(1+I / 2) /(1+I)^{2}$ is the so-called Stoner factor that relates to the onset of magnetic ordering; $f$ is the density of states at the Fermi level. Again, under conditions where either of the factors in the denominator becomes small, the paramagnetic susceptibility can greatly exceed that of ordinary metals. As has been established in early experiments [5], the susceptibility of metallic Ti-doped $\mathrm{V}_{2} \mathrm{O}_{3}$ is exceptionally large.

All of the above information attests to the importance of correlation effects. However, the physical interpretation of the properties of $\mathrm{V}_{2} \mathrm{O}_{3}$ is more complicated than this simple picture suggests. First, the theoretical analysis is based on a model in which electrons occupy a half filled nondegenerate band. As is discussed below, the actual situation is more complicated. Second, the effects of the lattice cannot be discounted: there is an enormous, $70 \mathrm{~K}$, hysteresis in the transition temperature of the PM-PI transition, even though the lattice symmetry remains unaltered; the transition to the PI state involves a volume increase of $1.4 \%$. The effect of the lattice on the transition has so far not been properly taken into account. Aside from the hysteresis, the lattice presumably plays an important role in the third PI-PM ${ }^{\prime}$ transition that occurs over a wide temperature range of 400-800 K, rather than as a first-order transformation. Lastly, magnetic interactions do play an important role, witness the fact that the molar entropy change in the PM-AFI transition very nearly conforms to the relation $\Delta S=R \ln (2 J+1)$ where $J$ is the total angular momentum of each cation. Despite these cautionary remarks, $\mathrm{V}_{2} \mathrm{O}_{3}$ is generally regarded as a paradigm of a Mott material undergoing metal-insulator transitions.

We now attempt to rationalize the reentrant metallic feature and the general phase diagram for the $\mathrm{V}_{2} \mathrm{O}_{3}$ system. This may be done on a very elementary basis by contrasting the properties of localized charge carriers occupying $N$ isolated degenerate energy levels (for the PI phase) with the characteristics of itinerant charge carriers in a half filled nondegenerate band (in the PM phase): We set the energy of the localized electrons as $E_{1}=0$. The entropy of each carrier is given by $S_{1} / N=-k_{\mathrm{B}} \ln 2$ because a given electron can occupy the localized state in either the spin-up or spin-down configuration. The free energy is then given by $F / N=\left(-k_{\mathrm{B}} \ln 2\right) T$; i.e., the free energy varies linearly with temperature.

For the itinerant case, we start with the Sommerfeld model for the electronic contribution to the heat capacity $C / N=\gamma T$. On performing an integration over temperature, one obtains the energy as $E_{i} / N=E_{0} / N+\frac{1}{2} \gamma T^{2}$, where $E_{0}$ is a constant. Similarly, dividing $C$ by $T$ and integrating yields the entropy $S_{i} / N=\gamma T$. 


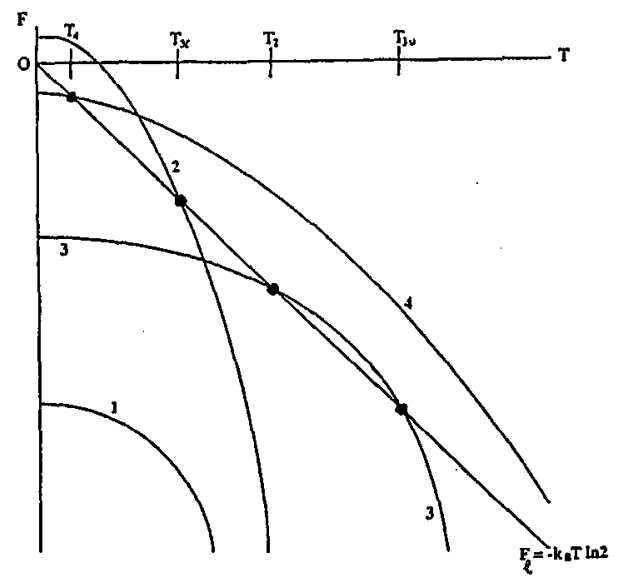

Fig. 2. Plots of free energy $F$ vs. temperature for localized electrons (straight line passing through the origin) and itinerant electrons(parabolas). See text for details.

Then the free energy reads

$$
F_{i} / N=E_{i} / N-T S_{i} / N=E_{0} / N-\frac{1}{2} \gamma T^{2} .
$$

Thus, $F_{1}$ changes parabolically with $T$. The results are displayed graphically in Fig. 2 as a straight line passing through the origin (localized electrons), and as a set of parabolas with different intercepts $E_{0}$ (itinerant charge carriers). Note the following: when $E_{0}$ is a large negative quantity, the parabola (curve 1) always remains below the straight line; i.e., the metallic PM phase has the lower free energy and therefore is stable over the entire temperature range. As $E_{0}$ becomes less negative, the parabola (curve 3 ) intersects the straight line twice: in the temperature ranges $T<T_{3 l}$ and $T>T_{3 u}$, the parabola represents the system of lower free energy. In the range $T_{3 l}<T<T_{3 u}$ the straight line represents the system of lower free energy. This immediately rationalizes the reentrant metallic behavior seen in the $\mathrm{V}_{2} \mathrm{O}_{3}$ system above the magnetic ordering temperature. If on the other hand, $E_{0}$ is slightly positive, the parabola (curve 2) intersects the straight line at the temperature $T_{2}$. For $T<T_{2}$ the localized system has the lower free energy and for $T>T_{2}$ the reverse holds. This rationalizes the common case where the insulating phase at low temperature yields to the metallic configuration higher $T$. Of relevance to the $\mathrm{NiS}_{2-x} \mathrm{Se}_{x}$ system discussed below is the case where $E_{0}$ is slightly negative (curve 4). Here the parabola lies below the straight line only at very low temperatures, above which the situation reverses. This represents the case of a metal at low temperature giving way to an insulating phase at high temperature.

It is evident that the nature of the experimental transitions depends markedly on the value of $E_{0}$. Two contributions make up this quantity in the simplest case. Relative to the localized electrons, the itinerant carriers have an average kinetic energy $-|\bar{\epsilon}|$ in the free state; correlation interactions are roughly accounted for through a function $\Phi(\eta)$ that is specified by the theory [7, 8]. This factor depends on the degree of double occupancy $\eta$. The second contribution involves the Coulomb potential $U$ between two electrons occupying a given lattice site with reversed spins: the total potential interaction energy is then given by $U \eta$. This 
leads to the simple relationship $E_{0}=-\Phi(\eta)|\bar{\epsilon}|+U \eta$. One sees that when $U \eta$ is small relative to $-\Phi|\bar{\epsilon}|, E_{0}$ has a large negative value, corresponding to the intercept for curve 1 in Fig. 2. When $U \eta$ becomes comparable to but remains smaller than $-\Phi|\bar{\epsilon}|, E_{0}$ moves up on the energy scale as shown for the intercept of curve 3 of Fig. 2. When the kinetic and potential energies are very nearly in balance one obtains curves 2 or 4 , depending on whether or not the potential energies slightly outweigh the kinetic contribution.

This very elementary analysis provides a qualitative explanation of the experimental findings in the $\mathrm{V}_{2} \mathrm{O}_{3}$ system. The actual situation is, of course, far more complicated. This is shown in recent work by Bao et al. [9] who have shown by neutron diffraction experiments that magnetic ordering effects persist not only in the AFM phase but also in the PM and PI phase to temperatures in excess of 20 times the ordering temperature. These magnetic correlations are short ranged and form a spin density spiral as shown in Fig. 3. Its existence has been attributed to the presence of nested Fermi surfaces displayed in a recent publication [10]. By contrast, the AFI phase exhibits the magnetic order displayed in Fig. 4; here slabs of ferromagnetically aligned spins along the $c$ direction alternate with slabs of spins aligned in the opposite direction [11]. The entire phase diagram is shown schematically in Fig. 5. The AFI alignment occurs only in the lower right hand

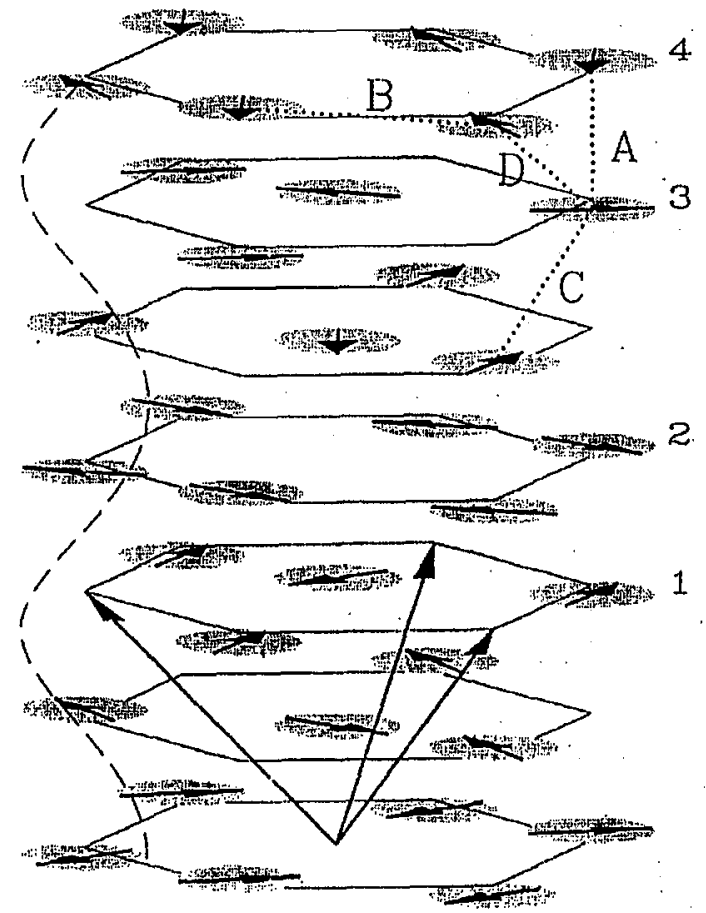

Fig. 3. Magnetic ordering of $\mathrm{V}$ spins in the spin density wave arrangement. 


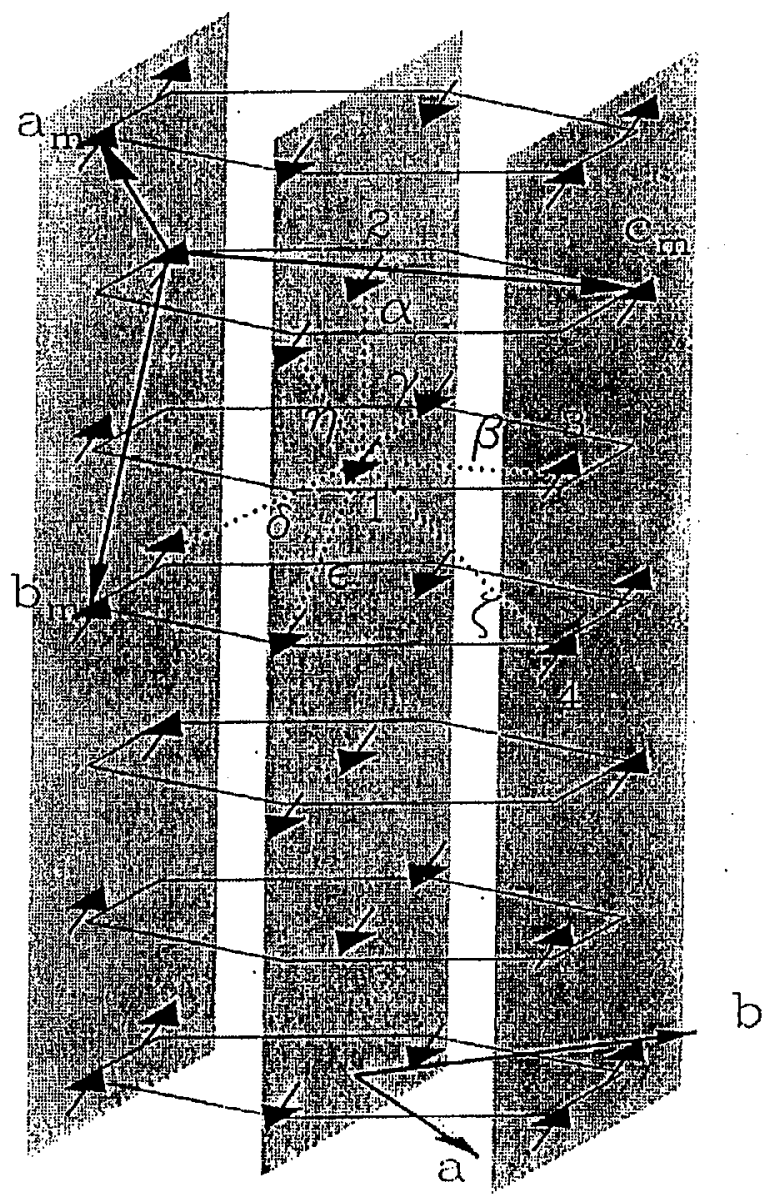

Fig. 4. Magnetic ordering of $\mathrm{V}$ spins for the AFI phase.

side corner and the spiral spin density waves are encountered only outside this region. The magnetic realignment engendered in the PM-AFI or PI-AFI phase transitions call into question the current use of a single Hamiltonian operator of the form proposed by Hubbard. Account should be taken of the nondegenerate singly occupied energy level $a_{1}$ and of the doubly degenerate levels $e_{\pi}$ and $e_{\pi}^{*}$, occupied by the second electron; this splitting of the original $t_{2 \mathrm{~g}}$ levels results from the action of the trigonal field of the corundum lattice. One must now invoke the orbital ordering effects concomitant to such energy splittings [12]. These are displayed in the inset to Fig. 5 and show the coupling of the orbital and spin ordering at $T=0$. With rising temperature, fluctuations in orbital occupancy produce corresponding spin fluctuations and disorder, as well as shortened correlation lengths. However, this explanation has recently been called into question by Sawatzky and co-workers [13] who proposed that all three of the $a_{1}, e_{\pi}$, and $e_{\pi}^{*}$ energy states experience comparable electron occupancies that change with temperature and composition. At the time of writing, this issue has not been resolved. 


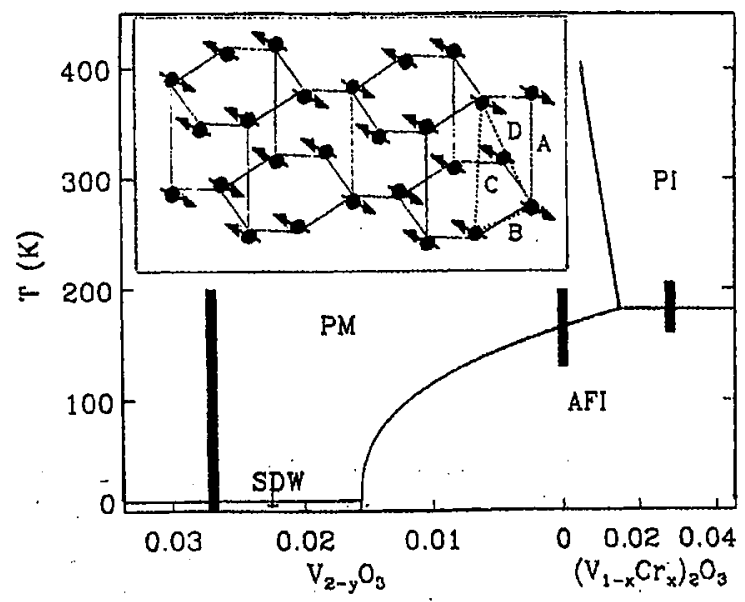

Fig. 5. Phase diagram for $\mathrm{V}_{2-y} \mathrm{O}_{3}$ and $\left(\mathrm{V}_{1-x} \mathrm{Cr}_{x}\right)_{2} \mathrm{O}_{3}$. Vertical bars indicate neutron diffraction data in experiments taken as a function of temperature for three compositions. Inset shows orbital ordering and spin ordering for the AFI phase.

Summarizing, $\mathrm{V}_{2} \mathrm{O}_{3}$ is a paradigm of a system in which electron correlation effects dominate the physical properties. However, as shown above, magnetic ordering phenomena and electron lattice interactions also play an important role. The situation is sufficiently complicated that at this time no final conclusions can be drawn, pending further experimental and theoretical developments.

\section{Properties of the $\mathrm{NiS}_{2-x} \mathrm{Se}_{x}$ system}

A second system of interest is the $\mathrm{NiS}_{2-x} \mathrm{Se}_{x}$ system: $\mathrm{NiS}_{2}$ is a good insulator whereas $\mathrm{NiSe}_{2}$ is a poor metal. The two end members can be mixed in all proportions; Vegard's law is accurately obeyed over the entire composition range $0 \leq x \leq 2$. By judicious admixture of $\mathrm{S}$ and $\mathrm{Se}$ one can therefore study the gradual transformation of the system from the metallic to the insulating state. The advantage of this system over almost all others is that all compositional alterations involve only the anion sublattice, while keeping the cation sublattice intact. Moreover, since $\mathrm{S}$ and $\mathrm{Se}$ are in the same column of the periodic table, the electron count remains unaltered in the substitution process.

Experimental resistivity measurements on single crystal $\mathrm{NiS}_{2-x} \mathrm{Se}_{x}$ for $x \leq 0.7$ are shown in Fig. 6, and the corresponding Seebeck coefficient measurements are shown in Fig. 7 [14]. One may distinguish three regimes. For $0 \leq x \leq 0.3$ the resistivity drops essentially monotonically with rising temperature, as shown in Fig. 6a. The two exponential decays are associated with activation energies of 330 and $110 \mathrm{meV}$, separated by a range of nearly constant resistivity. For compounds with $0.38 \leq x \leq 0.55$, the material is metallic at low temperatures and experiences a transition to the insulating state somewhere in the range 30 to $80 \mathrm{~K}$. For $x=0.38$, the low temperature, $T$ independent resistivity has the extraordinarily high value of $140 \Omega \mathrm{cm}$, which represents one of the highest reported metallic resistivities, a full eight orders of magnitude larger than those of commercial copper. 

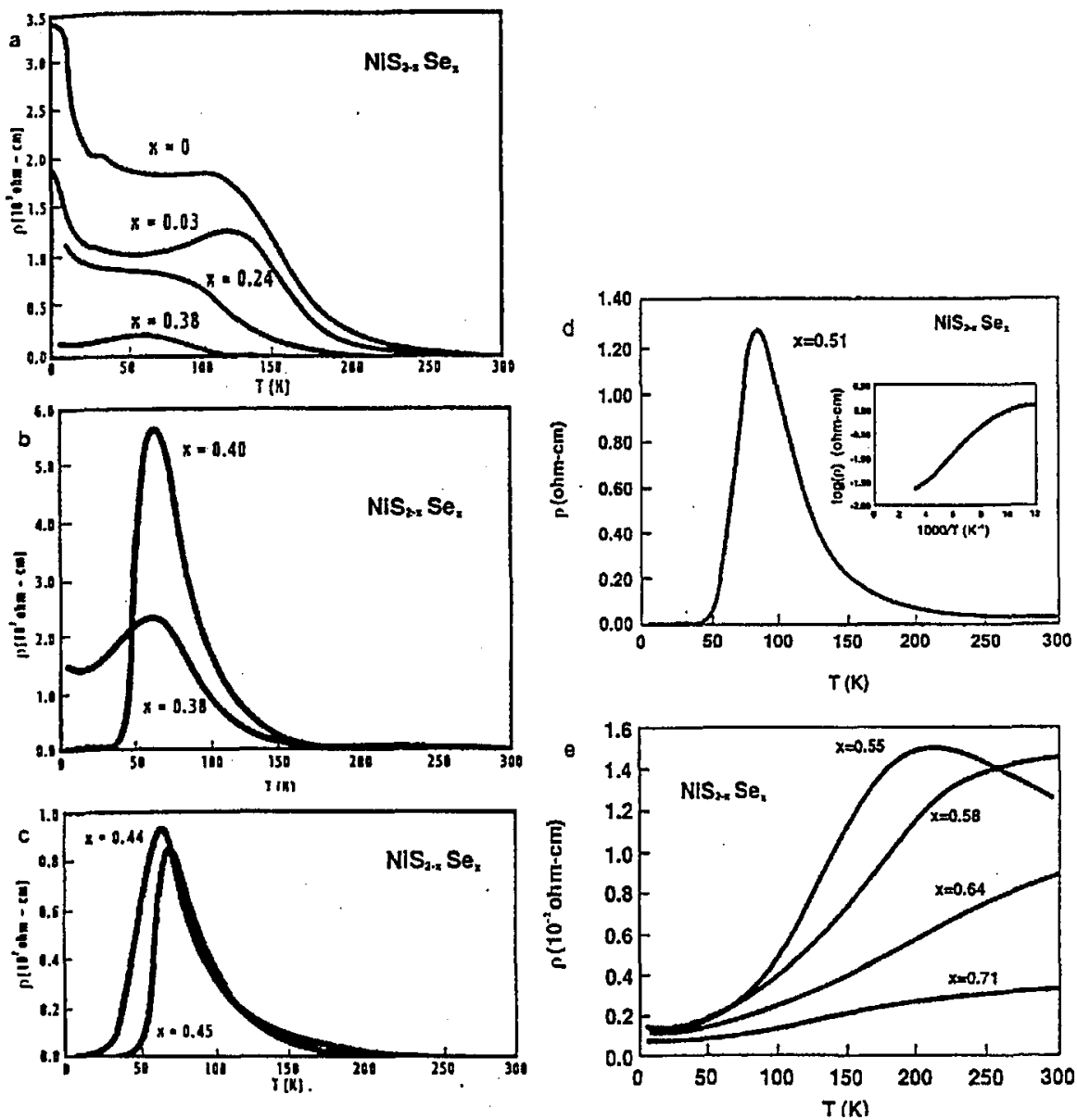

Fig. 6. Electrical resistivities for $\mathrm{NiS}_{2-x} \mathrm{Se}_{x}$ single crystals for various $x$ as a function of temperature. See text for details.

These very high resistivities, as well as the corresponding electronic contributions to the specific heat and the measured magnetic susceptibilities [14], are consistent with the electron correlation effects anticipated for this composition range. The AFM-PI transition with rising temperature can therefore be rationalized as described in conjunction with curve 4 of Fig. 2. That is, due to correlation effects among electron a gap opens up in the density-of-states when the temperature is raised. This has been verified in electron tunnelling studies reported recently [15].

In the range $x>0.55$, the material gradually becomes more metallic with increasing Se content, as shown in Fig. 6e; there is no further dramatic change in the resistivity pattern up to the end member $\mathrm{NiSe}_{2}$ [16].

The thermoelectric data present an interesting puzzle: precisely in the temperature range where the alloys with $0 \leq x \leq 0.30$ show a rapid rise in resistivity as $T \rightarrow 0$, the Seebeck coefficient $\alpha$ almost vanishes; this runs counter the generally accepted rule that a rise in $\rho$ should be mirrored by a corresponding increase 

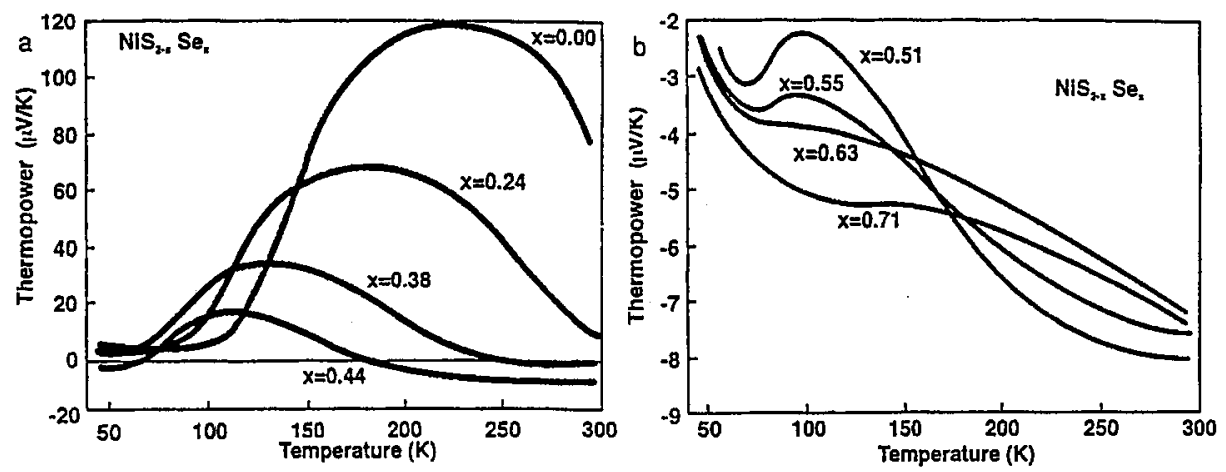

Fig. 7. Seebeck coefficients for $\mathrm{NiS}_{2-x} \mathrm{Se}_{x}$ single crystals. Note the greatly expanded abscissa scale for the metallic phase.

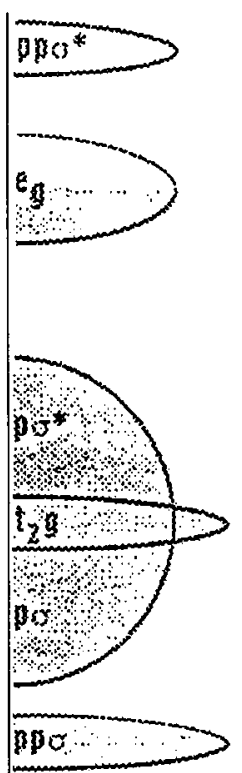

[a]

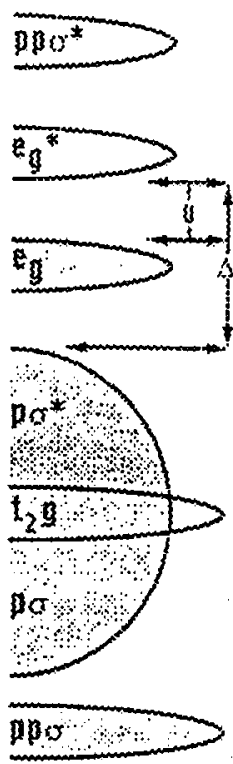

(b)
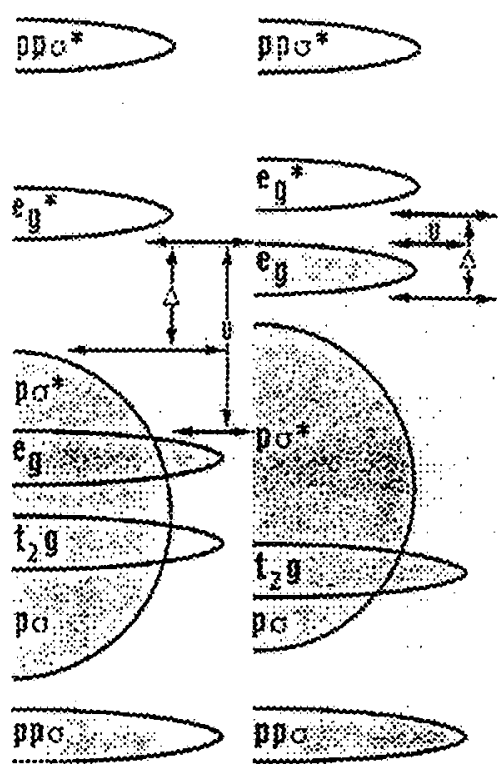

[c]

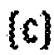

Fig. 8. Proposed band structure diagram for $\mathrm{NiS}_{2-x} \mathrm{Se}_{x}$. See text for details.

in $\alpha$. Further, at higher temperatures, where $\rho$ decreases with rising $T, \alpha$ passes through a pronounced maximum shown in Fig. $7 \mathrm{a}$. This again runs counter the usual experimental situation. It is only in the metallic range $x>0.51$ where $\alpha$ changes linearly with $T$ in the expected manner; on the very greatly expanded abscissa scale of Fig. $7 \mathrm{~b}$ the deviations from linearity are greatly exaggerated.

The thermopower data may be rationalized on the basis of the proposed band structure diagram shown in Fig. 8 [2]. We show the overall disposition of bands close to the Fermi level: The $p p \sigma$ and $p p \sigma^{*}$ narrow bands represent the bonding 
and antibonding configurations of the $(S S)^{-2}$ unit, augmented by bonding to the $e_{\mathrm{g}}$ state of $\mathrm{Ni}$. The $p \sigma$ and $p \sigma^{*}$ parts represent the energy of primarily $3 p$ character for sulfur/selenium. The narrow $t_{2 \mathrm{~g}}$ bands represent $\mathrm{Ni}$ states that bond only weakly to anionic neighbors. Lastly, we show schematically the half filled band of $e_{\mathrm{g}}$ states with some anionic admixture in the absence of electron correlations. As is well-established, for such half filled bands, correlation effects are expected to split this band into subbands. One may then encounter the Mott-Hubbard configuration depicted in part (b), where both halves of the $e_{\mathrm{g}}$ band are shown as lying above the $3 p \sigma$ states; the intraatomic repulsion energy $U$ is smaller than the energy difference $\Delta$ between the top of the $3 p \sigma^{*}$ and the bottom of the $e_{\mathrm{g}}^{*}$ bands. This is a manifestation of the well-known Mott-Hubbard metal-insulator representation arising from electron correlations. This split band structure immediately rationalizes the fact that $\mathrm{NiS}_{2}$ is a good insulator at low temperatures. However, an alternative disposition is shown in part (c), where $\Delta<U$, so that thermal excitations of electrons occur across the gap between the $3 p \sigma^{*}$ and $e_{\mathrm{g}}$ states. This is a manifestation of a charge transfer insulator of the type described by Zaanen et al. [17]. Opinion is divided as to which category is appropriate for $\mathrm{NiS}_{2}$. Bocquet and co-workers [18] strongly argue in favor of the latter alternative, but their evidence is indirect and not in accord with thermoelectric effects described above. Lastly, in part (d) we depict the case where $\Delta$ slightly exceeds $U$, such that at low temperatures the compound is a Mott-Hubbard insulator and at higher temperature the charge transfer effects become manifest. We believe that this representation is appropriate to the $\mathrm{NiS}_{2-x} \mathrm{Se}_{x}$ system for $x<0.44$, as will shortly be documented.

Of immediate relevance is the band structure shown in Fig. 8a that presumably applies to alloys with $x>0.55$. On the other hand, for alloys with $x<0.38$, the situation depicted in Fig. 8d is believed to hold, with $\Delta$ only slightly exceeding $U$, so that the upper band edges of the $e_{\mathrm{g}}$ and $p \sigma^{*}$ bands very nearly coincide. In this scenario, the Seebeck coefficient at very lowest temperatures involves contributions from electrons thermally promoted across the Hubbard gap to the $e_{\mathrm{g}}^{*}$ states, as well as from holes left in the lower $e_{\mathrm{g}}$ subband. The overall Seebeck coefficient is then given by

$$
\alpha=\frac{-\sigma_{n}\left|\alpha_{n}\right|+\sigma_{p} \alpha_{p}}{\sigma_{n}+\sigma_{p}},
$$

where $\sigma_{n}, \sigma_{p}$ are the partial conductivities of electrons and holes and $\alpha_{n}, \alpha_{p}$ are the corresponding contributions to the Seebeck coefficient. The expression shows that for mirror image bands the electron and hole contributions nearly cancel out, so that the Seebeck coefficient should be very small, precisely in the regime where the overall resistivity rises steeply with falling $T$, as fewer and fewer carriers remain in the $e_{\mathrm{g}}^{*}$ subband. However, as the temperature is increased, electrons from the much wider $p \sigma^{*}$ band begin to be thermally promoted as well. The resulting holes in the $p \sigma^{*}$ band are very mobile and tend to swamp out the contributions of charge carriers in the much narrower $e_{\mathrm{g}}$ and $e_{\mathrm{g}}^{*}$ bands. The alloy thus gradually assumes the characteristics of a one-band $p$ type semiconductor with a very large positive Seebeck coefficient; $\alpha$ rises sharply with $T$. With a further rise in $T$, an ever larger number of mobile holes is produced and the material then becomes a degenerate 
semiconductor for which $\alpha$ diminishes with rising $T$. This rationalizes the large peak in the observed Seebeck coefficients at more elevated temperatures. Further work is needed to check whether the proposed simplistic model should be modified.

\section{Transitions of variable order in magnetite}

Another example of considerable interest is the Verwey transition in magnetite, $\mathrm{Fe}_{3} \mathrm{O}_{4}$. By now approximately 900 papers have been devoted to this subject. Most of the earlier work is of dubious value because it had not been appreciated that extreme precautions must be taken to fix the oxygen/metal ratio in this compound in order to obtain reproducible results. Procedures for appropriate sample treatments are published elsewhere [19].

Heat capacity data on properly annealed stoichiometric specimens [20] show a very sharp spike at the transition temperature of $T_{v}=121 \mathrm{~K}$. The first order transition corresponds to an entropy $\Delta S_{v}=R \ln 2$ per mole of $\mathrm{Fe}_{3} \mathrm{O}_{4}$. With increasing $\delta$ in $\mathrm{Fe}_{3(1-\delta)} \mathrm{O}_{4}$ (i.e., when excess oxygen is incorporated in the lattice) the transition temperature diminishes, as does $\Delta S_{v}$. Beyond a critical concentration $\delta_{\mathrm{c}}=0.0039$, one encounters instead a lambda-type specific heat anomaly, indicative of a second or higher order transition. $T_{v}$ decreases further with rising $\delta$ up to the phase boundary where hematite is formed. We designate magnetite undergoing first or higher order transitions as compounds of type I or II.

This change in the nature of the phase transition is also reflected in electrical properties. As an example, we show in Fig. 9 the dependence of resistivity $\rho$ on temperature for $\mathrm{Zn}$-doped $\mathrm{Fe}_{3} \mathrm{O}_{4}, \mathrm{Fe}_{3-x} \mathrm{Ti}_{x} \mathrm{O}_{4}$, whose electrical properties are virtually identical to those of $\mathrm{Fe}_{3(1-\delta)} \mathrm{O}_{4}$ with $x=3 \delta$. One can clearly discern the discontinuity in $\rho$ at $T_{v}$ for type I specimens, as distinct from the smoother variation for type II materials. Comparable distinctions occur in Seebeck coef-

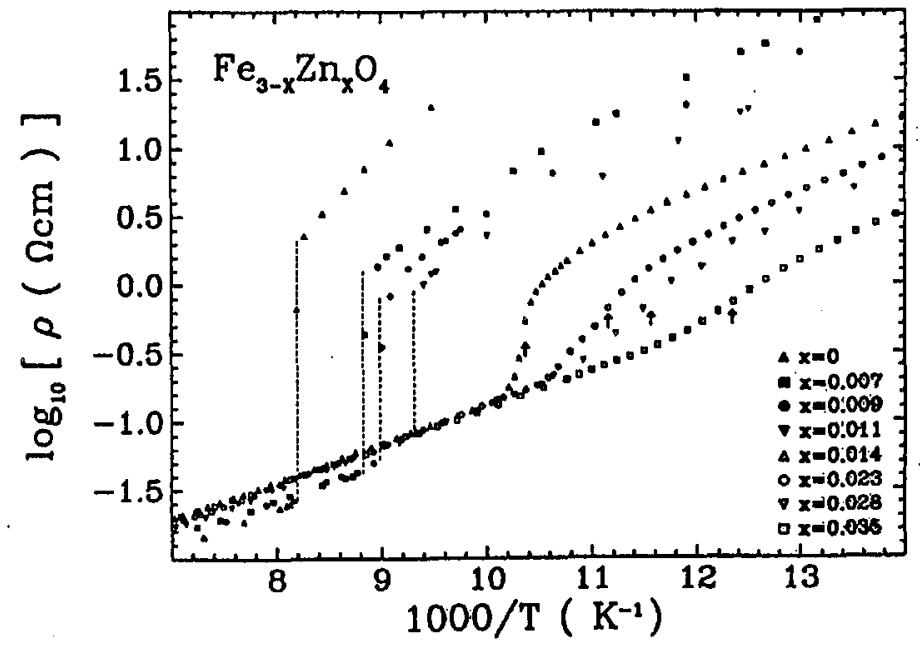

Fig. 9. Electrical resistivity for single crystal $\mathrm{Fe}_{3-x} \mathrm{Zn}_{x} \mathrm{O}_{4}$ for various $x$ as a function of temperature. 


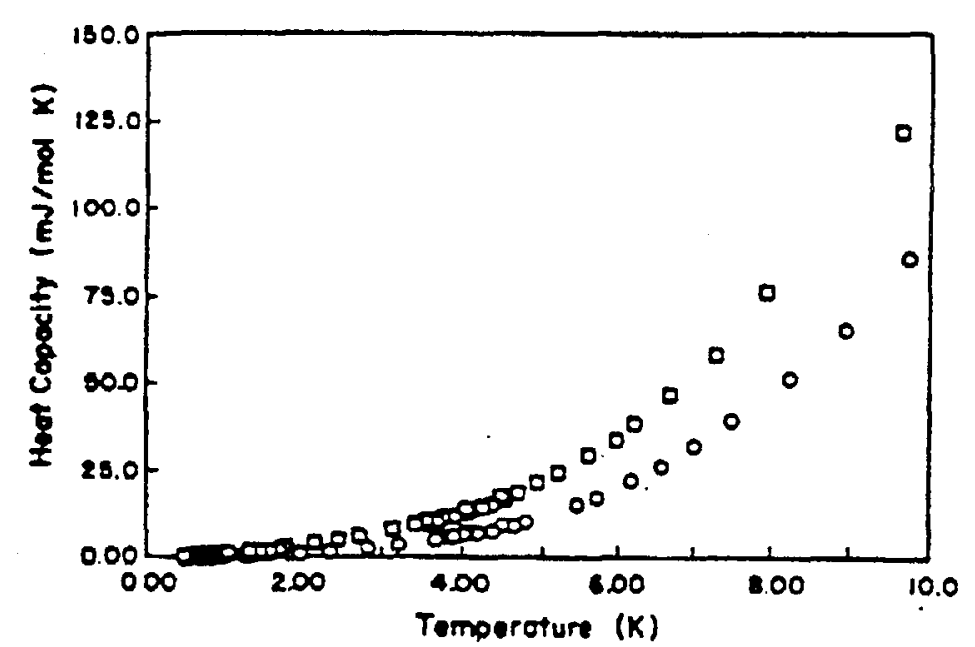

Fig. 10. Heat capacity of single crystal $\mathrm{Fe}_{3(1-\delta)} \mathrm{O}_{4}$. Open circles and squares represent data for $\delta=0$ and $\delta=0.0049$ taken at low temperature. Note the difference for $I$ and II-type specimens.

ficient measurements [21]. One should also note that for $x>0.036, \mathrm{Fe}_{3-x} \mathrm{Ti}_{x} \mathrm{O}_{4}$ undergoes no transition; thus, one is actually dealing with three types of magnetite.

Not only electrical characteristics but also the lattice properties of $\mathrm{Fe}_{3-x} \mathrm{Ti}_{x} \mathrm{O}_{4}$ are altered at the transition. The heat capacities of type I and II materials differ significantly below their respective $T_{v}$ values down to the lowest measured temperatures, as shown in Fig. $10[20,22]$. This trend is also mirrored in the Debye $\Theta$ values extracted from such measurements. Below $T_{v}$, the $\Theta$ values increase dramatically for type I samples, whereas they extrapolate smoothly across the transition for second order specimens [23]. The change in lattice properties of $\mathrm{Fe}_{3-y} \mathrm{Ti}_{y} \mathrm{O}_{4}$ and of $\mathrm{Fe}_{3-x} \mathrm{Zn}_{x} \mathrm{O}_{4}$ is also documented in recent careful neutron diffraction studies of the change from spinel to monoclinic structure as $T$ is lowered past $T_{v}$ [24].

The distinction between the two types of transition is further emphasized in Fig. 11 showing plots of $T_{v}$ versus compositions of $\mathrm{Fe}_{3(1-\delta)} \mathrm{O}_{4}, \mathrm{Fe}_{3-y} \mathrm{Ti}_{y} \mathrm{O}_{4}$, and $\mathrm{Fe}_{3-x} \mathrm{Zn}_{x} \mathrm{O}_{4}$. With the correspondence $x=y=3 \delta$, the regimes $\mathrm{I}$ and $\mathrm{II}$ are very clearly in evidence. Beyond the merging point of the two straight lines no transition is observed.

A very simplistic model of the Verwey transition is offered below. Again, it must be supplemented by more sophisticated approaches detailed elsewhere [25]. By way of introduction, we note that the electrical characteristics arise from electrons that hop from one octahedrally coordinated $\mathrm{Fe}^{2+}$ ion to an adjacent $\mathrm{Fe}^{3+}$ ion similarly coordinated. Among the collection of iron cations in octahedral coordination one therefore encounters nearest neighbor pairs in the $\mathrm{Fe}^{3+}-\mathrm{Fe}^{3+}\left(\mathrm{Fe}^{3+}-\mathrm{Fe}^{2+}, \mathrm{Fe}^{2+}-\mathrm{Fe}^{3+}\right)$, and $\mathrm{Fe}^{2+}-\mathrm{Fe}^{2+}$ configurations. This renders plausible the scheme of replacing magnetite by collections of the indicated site pairs. These are associated with energies $\epsilon_{0}, 2 \epsilon_{1}, \epsilon_{2}$ and probabilities $\beta_{0}, 2 \beta_{1}, \beta_{2}$ respectively. 


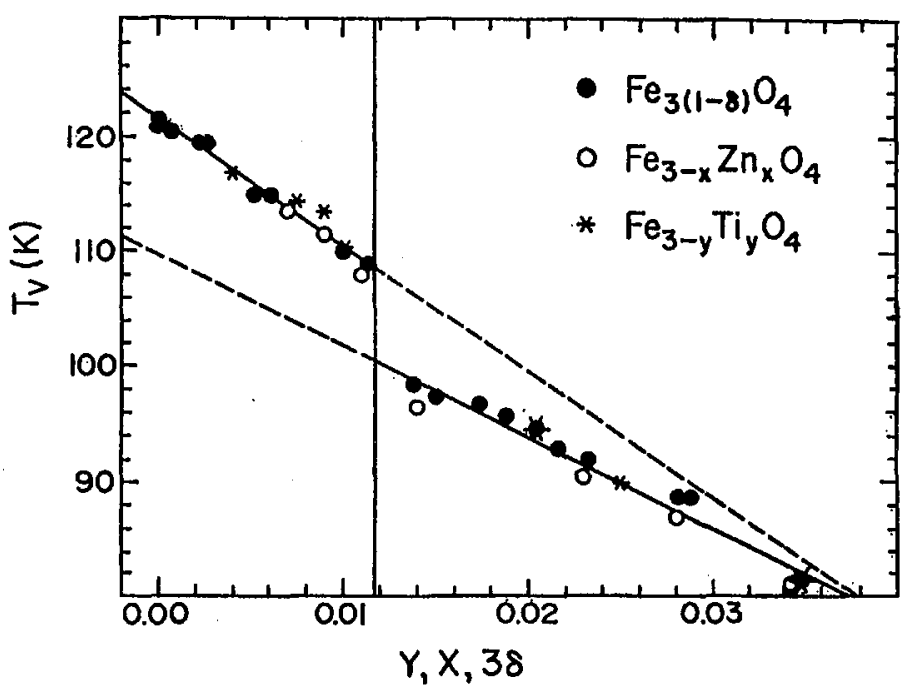

Fig. 11. Variation of Verwey transition temperature with composition for first and second order regimes in nonstoichiometric $\mathrm{Zn}$-doped or Ti-doped magnetite.

At an extremely elementary level the statistical treatment developed by Spałek, Honig, and collaborators [25] may be summarized as follows: The energy of the system is given by $E / N=\beta_{0} \epsilon_{0}+2 \beta_{1} \epsilon_{1}+\beta_{2} \epsilon_{2}$, where $N$ is the number of octahedral lattice sites. The entropy is specified by $S / N=-k_{\mathrm{B}}\left(\beta_{0} \ln \beta_{0}+2 \beta_{1} \ln \beta_{1}+\right.$ $\beta_{2} \ln \beta_{2}$ ). Lastly, the various probabilities must add up to unity: $\beta_{0}+2 \beta_{1}+\beta_{2}=1$. The free energy per site then becomes

$$
\begin{gathered}
F / N=E / N-T S / N=\beta_{0} \epsilon_{0}+2 \beta_{1} \epsilon_{1}+\beta_{2} \epsilon_{2} \\
+k_{B} T\left(\beta_{0} \ln \beta_{0}+2 \beta_{1} \ln \beta_{1}+\beta_{2} \ln \beta_{2}\right) .
\end{gathered}
$$

We now simplify, noting that the $\mathrm{Fe}^{2+}-\mathrm{Fe}^{2+}$ site pairing is unlikely because of the strong repulsion between electrons on adjacent sites. We take the extreme limit of setting $\beta_{2}=0$, thereby obtaining $\beta_{0}=1-2 \beta_{1}$. Next, we introduce the order parameter (fraction of thermally promoted electrons) as $\psi=2 \beta_{1}$. We further assume that the energy difference $\epsilon_{1}-\epsilon_{0}$ varies with $\psi$ as $\epsilon_{1}-\epsilon_{0}=\epsilon-(\lambda / 2) \psi$. This reflects the fact that as particles are thermally promoted from the filled ground state of energy $\epsilon_{0}$, to the excited state at energy $\epsilon_{1}$, there is an increasing energy of attraction between the promoted electrons in the excited state and the holes left behind, proportional to the degree of occupation of the former. The factor $\lambda / 2$ is used for later notational convenience. The free energy may then be rewritten as

$$
\begin{aligned}
F / N & =\left(1-2 \beta_{1}\right) \epsilon_{0}+2 \beta_{1} \epsilon_{1}+k_{\mathrm{B}} T\left[\left(1-2 \beta_{1}\right) \ln \left(1-2 \beta_{1}\right)+\beta_{1} \ln \beta_{1}\right] \\
& =\epsilon \psi-\frac{1}{2} \lambda \psi^{2}+k_{\mathrm{B}} T[(1-\psi) \ln (1-\psi)+\psi \ln \psi-\psi \ln 2] .
\end{aligned}
$$

To enforce equilibrium we minimize $F$ with respect to $\psi$ : from $\partial F / \partial \psi=0$ we obtain the equation of state

$$
(\epsilon-\lambda \psi) / k_{\mathrm{B}} T=\ln [(1-\psi) / \psi]+\ln 2,
$$




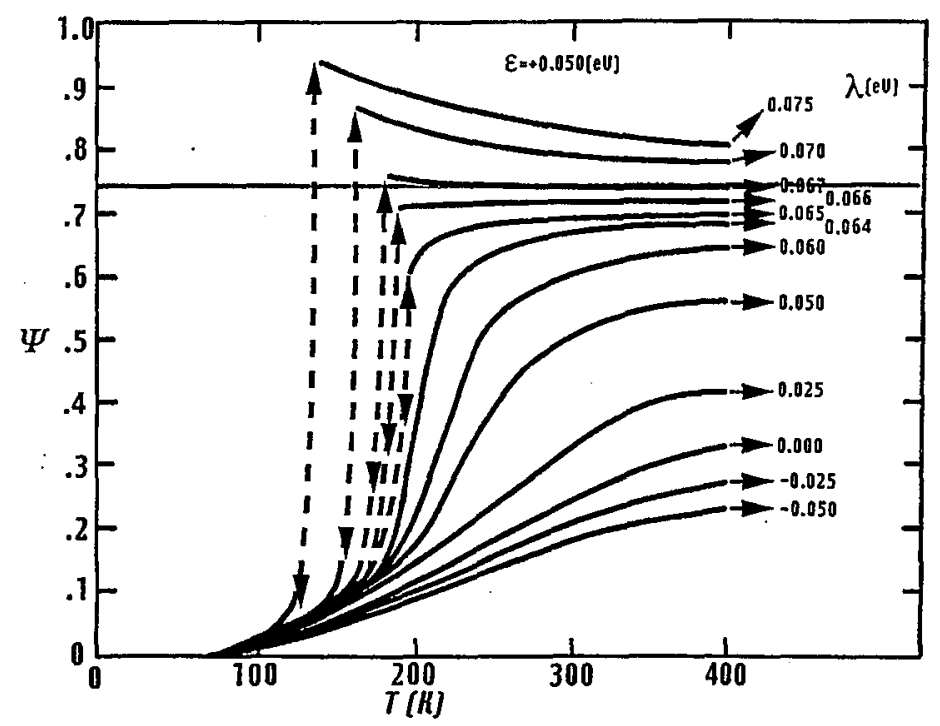

Fig. 12. Variation of order parameter with reduced temperature for several values of $\epsilon / \lambda$, as calculated from Eq. (5).

which must be solved numerically to determine how $\psi$ varies with $T$ for a given set of assumed values of $\epsilon$ and $\lambda$. A generic calculation is shown in Fig. 12 as a plot of $\psi$ vs. $k_{\mathrm{B}} T / \lambda$ for various $\epsilon / \lambda$ values in the range -1 (bottom) to $2 / 3$ (top). Note that for the larger $\epsilon / \lambda$ the order parameter exhibits a discontinuity that becomes progressively smaller as $\epsilon / \lambda$ drops. Beyond a critical $\epsilon / \lambda$ the variation of $\psi$ with $k_{\mathrm{B}} T / \lambda$ becomes continuous. This is symptomatic of a change from a first order transformation to a crossover regime. The present theory should therefore be useful in the interpretation of the experimental findings in the $\mathrm{Fe}_{3} \mathrm{O}_{4}$ system. That this is the case for heat capacity and electrical characteristics of pure magnetite, or for $\mathrm{Fe}_{3} \mathrm{O}_{4}$ doped with oxygen, $\mathrm{Zn}, \mathrm{Al}$, or $\mathrm{Ti}$ has been well documented in the literature; the subject has been reviewed elsewhere [26].

A somewhat different mathematical analysis with slightly different starting assumptions results in an equation of state like Eq. (5), with the $\ln 2$ term missing. The resulting relation corresponds to a standard second order transformation. Experimentally, it may be very difficult to distinguish between the crossover and the second order transition regime. In fact, recent neutron diffraction measurements raise questions as to whether a second order transformation exists at all or whether the higher order transformation involves formation of domains [27]; this question deserves further study. However, the mathematical model proposed here nicely accounts for a variety of experimental data after the theory has been properly parametrized [25]: The variation of $\Delta S_{v}$ with $\delta$ in $\mathrm{Fe}_{3(1-\delta)} \mathrm{O}_{4}$ is also correctly reproduced, as are the Seebeck coefficient measurements and the observed resistivities $[26,28]$. In this sense the elementary approach to the Verwey transition problem is successful. A more sophisticated approach yields very similar results [29]. 


\section{Conclusion}

In this article we have cited several examples of materials that undergo various types of transitions that may be associated with electron correlation phenomena. This is still a very active field of endeavor; considerable further work is needed to clarify the outstanding issues mentioned above.

\section{Acknowledgments}

The author wishes to express his indebtedness to Prof. J. Spałek for his active participation in the interpretation of the experimental work and for many fruitful discussions concerning the theoretical aspects. He similarly indebted to all collaborators for their patient accumulation of the experimental data. This work was supported on NSF grant DMR96-12130.

\section{References}

[1] J.M. Honig, J. Spałek, Solid State Physics 2, Eds. M.A.K.L. Dissanayake, K. Tenakone, D.A. Ileperuma, Vol. 8, Nova Science, Commack, NY 1991, p. 1; M. Yethiraj, J.M. Honig, in: Advances in the Synthesis and Reactivity of Solids, Ed. T.E. Mallouk, Vol. 2, JAI Press, London 1994, p. 235; J.M. Honig, Acta Phys. Pol. A 85, 53 (1994); J. Spałek, J. Solid State Chem. 88, 70 (1990); M. Yethiraj, J. Solid State Chem. 88, 53 (1990).

[2] J.M. Honig, J. Spałek, Chem. Mater. 10, 2910 (1998); J.M. Honig, Proc. Indian Acad. Sci. (Chem. Sci.) 96, 39 (1986); J.M. Honig, J. Spałek, J. Less.-Common Met. 156, 423 (1989).

[3] H. Kuwamoto, J.M. Honig, J. Appel, Phys. Rev. B 22, 2626 (1980).

[4] S.A. Shivashankar, J.M. Honig, Phys. Rev. B 28, 5695 (1983).

[5] D.B. McWhan, A. Menth, J.P. Remeika, W.F. Brinkman, T.M. Rice, Phys. Rev. B 7, 1920 (1973); D.B. McWhan, J.P. Remeika, Phys. Rev. Lett. 27, 941 (1971); D.B. McWhan, T.M. Rice, Phys. Rev. Lett. 22, 887 (1969).

[6] Y. Ueda, K. Kosuge, S. Kachi, T. Takada, J. Phys. (Paris) C 40, 275 (1979); Y. Ueda, K. Kosuge, S. Kachi, J. Solid State Chem. 31, 171 (1980).

[7] J. Spałek, A. Datta, J.M. Honig, Phys. Rev. Lett. 59, 728 (1987); J. Spałek, M. Kokowski, J.M. Honig, Phys. Rev. B 39, 4175 (1989).

[8] J. Spałek, A.M. Oleś, J.M. Honig, Phys. Rev. B 28, 6802 (1983).

[9] W. Bao, C. Broholm, G. Aeppli, P. Dai, J.M. Honig, P. Metcalf, Phys. Rev. Lett. 78, 507 (1997); W. Bao, C. Broholm, G. Aeppli, S.A. Carter, P. Dai, C.D. Frost, J.M. Honig, P. Metcalf, J. Magn. Magn. Mater. 177-181, 283 (1998); W. Bao, C. Broholm, G. Aeppli, S.A. Carter, P. Dai, T.F. Rosenbaum, J.M. Honig, P. Metcalf, S.F. Trevino, Phys. Rev. B 58, 12727 (1998); W. Bao, C. Broholm, J.M. Honig, P. Metcalf, S.F. Trevino, Phys. Rev. B 54, R3726 (1996).

[10] T. Wolenski, M. Grodzicki, J. Appel, Phys. Rev. B 58, 303 (1998).

[11] R.M. Moon, Phys. Rev. Lett. 25, 527 (1970).

[12] T.M. Rice, in: Spectroscopy of Mott Insulators and Correlated Metals, Eds. A. Fujimori, Y. Tokura, Springer, New York 1995, p. 221; C. Castellani, C.R. Natoli, J. Ranninger, Phys. Rev. B 18, 4945, 4967, 5001 (1978); S. Inagaki, J. Phys. Soc. Jpn. 39, 596 (1975); K.I. Kugel, D.I. Khomskii, Sov. Phys.-JETP Lett. 15, 446 (1972); Sov. Phys.-JETP 37, 725 (1973); Solid State Commun. 13, 763 (1973); M. Cyrot, C. Lyon-Caen, J. Phys. (Paris) 36, 253 (1975). 
[13] G. Sawatzky, private communication and preprint.

[14] X. Yao, J.M. Honig, T. Hogan, C. Kannewurf, J. Spałek, Phys. Rev. B 54, 17469 (1996); X. Yao, S. Ehrlich, G. Liedl, T. Hogan, C. Kannewurf, J.M. Honig, Mater. Res. Soc. Symp. Proc. 453, 291 (1997).

[15] J.G. Rodrigo, S. Vieira, P. Somasundaram, J.M. Honig, F.A. Chudnovskii, V.N. Andreev, Phys. Rev. B 58, 10256 (1998).

[16] M. Kamada, M. Môri, T. Mitsui, J. Phys. Soc. C, Solid State Phys. 10, L643 (1977); S. Miyasaka, H. Tagaki, Y. Sekine, H. Takahashi, N. Môri, R.J. Cava, private communication.

[17] J. Zaanen, G.A. Sawatzky, J.W. Allen, Phys. Rev. Lett. 55, 418 (1995).

[18] A.E. Bocquet, K. Mayima, T. Mizokawa, A. Fujimori, T. Miyadai, H. Takahashi, M. Môri, S. Suga, J. Phys., Condens. Matter 8, 2389 (1996).

[19] J.P. Shepherd, R. Aragón, J.W. Koenitzer, J.M. Honig, Phys. Rev. B 32, 1818 (1985); J.P. Shepherd, J.W. Koenitzer, R. Aragón, J. Spałek, J.M. Honig, Phys. Rev. $B$ 43, 8461 (1991).

[20] J.P. Shepherd, J.W. Koenitzer, R. Aragón, J. Spałek, J.M. Honig, Phys. Rev. B 43, 8461 (1991) and references therein.

[21] R.J. Rasmussen, R. Aragón, J.M. Honig, J. Appl. Phys. 61, 4395 (1987).

[22] J.W. Koenitzer, P.H. Keesom, J.M. Honig, Phys. Rev. B 39, 6231 (1989).

[23] A. Kozłowski, Z. Kąkol, D. Kim, R. Zalecki, J.M. Honig, Z. Anorg. Allg. Chem. 623, 115 (1997); A. Kozłowski, Z. Kąkol, D. Kim, R. Zalecki, J.M. Honig, Phys. Rev. B 54, 12093 (1996); A. Kozłowski, Z. Kąkol, R. Zalecki, K.S. Knight, J.M. Honig, J. Phys. (France) 7, C1-59 (1993).

[24] Z. Kąkol, A. Kozłowski, R. Zalecki, K. Knight, D. Kim, J.M. Honig, Electron Technol. 31, 22 (1998); A. Kozłowski, Z. Kakol, R. Zalecki, K.S. Knight, J. Sabol, J.M. Honig, J. Phys., Condens. Matter 11, 2749 (1999).

[25] R. Aragón, J.M. Honig, Phys. Rev. B 37, 209 (1988); J.M. Honig, J. Spałek, J. Less-Common Met. 156, 423 (1989); J.M. Honig, J. Spałek, J. Solid State Chem. 96, 115 (1992); J.M. Honig, J. Alloys Comp. 229, 24 (1995); H. Kloor, J.M. Honig, $J$. Solid State Chem., in press.

[26] R. Aragón, J.M. Honig, Phys. Rev. B 37, 209 (1988); J.M. Honig, Phys. Chem. Miner. 15, 476 (1988); J.M. Honig, J. Alloys Comp. 229, 24 (1995).

[27] R. Aragón, P.M. Gehring, S.M. Shapiro, Phys. Rev. Lett. 70, 1635 (1993);

R. Aragón, J.W. Koenitzer, J. Solid State Chem. 113, 225 (1994).

[28] D. Kim, J.M. Honig, Phys. Rev. B 49, 4438 (1994).

[29] H. Kloor, Ph.D. thesis, Purdue University, 1995, unpublished. 